\title{
KNOWLEDGEbASE SySTEMS IN NEURO SCIENCE - A STUDY
}

\author{
Dr. D.K. Sreekantha ${ }^{1}$, T.M. Girish ${ }^{2}$ and Dr. R.V.Kulkarni ${ }^{3}$ \\ ${ }^{1}$ Department of Computer Science \& Engineering, NMAMIT, Nitte, Karnataka \\ ${ }^{2}$ Dept. of CS, Basaveshwar Science College, Bagalkot, Karnataka \\ ${ }^{3}$ Head, Dept. of Computer Studies, CSIBER, Kolhapur, Maharastra
}

\begin{abstract}
The improvement of health and nutritional status of the society has been one of the thrust areas for social developments programmes of the country. The present states of healthcare facilities in India are inadequate when compared to international standards. The average Indian spending on healthcare is much below the global average spending. Indian healthcare Industry is growing at the rapid pace of more than 18\%, the fastest in the world. The prospects for Indian healthcare are to the tune of USD 40 billion, while global market is USD 1660 trillion. India has all the prospects to become medical tourism destination of the world, because it has a large pool of low-cost scientifically trained technical personal and is one of the favoured counties for cost effective healthcare. As per the reports of Global Burden of Neurological Disorders Estimations and Projections survey there is big shortage of neurologist in India and around the world. So Authors would like to develop an innovative IT based solution to help doctors in rural areas to gain expertise in Neuro Science and treat patients like expert neurologist. This paper aims to survey the Soft Computing techniques in treating neural patient's problems used throughout the world
\end{abstract}

\section{KEYWORDS}

Neurology, Expert System, Soft Computing

\section{INTRODUCTION}

India is one of the favoured countries for cost effective healthcare and has all the potential to become medical tourism destination of the world. The hospital and nursing home industry is growing at rate of $20 \%$ annually. India is one of the top 3 countries, where companies plan to spend most research and development funds over the next 3 years. The Indian clinical community is populated with English speaking, western-trained graduates. Two thirds of healthcare spending to the tune of USD 20 billion is out of pocket in private spending. India has 280 million strong middle and upper middle class population with 10-12 million high income groups, which could afford the lifestyle of their western counterparts. (Source : Economist Intelligence Unit, World Industry Outlook - Healthcare and Pharmacuticals, 2010 CAGR Compound annual growth rate). According to AAN2010 Practice Profile Form neurologists have devoted on average 42.3 hours a week in practice 2010. The number of visits to hospital has increased to 81.8 on average per week in 2010. These facts will signify the need for neurologist services in developing country like India around the world. Hence the researchers are focusing on building an innovative IT based solution to help novice doctors to treat neural patients in rural areas. During the last decade much research effort has been devoted to the development of expert systems to cope with complex medical decision-making. 
An expert system is "an embodiment within a computer of a knowledge-based component, from an expert skill, in such a form that the system can offer intelligent advice or make an intelligent decision about a processing function." such a system uses expert knowledge to attain high levels of performance in a narrow problem area. In the process of diagnosis, neurologists collect historical data, neurologic signs, and symptoms to arrive at a "best guess" as to stroke type, which then forms the basis for performing further diagnostic procedures such as computed tomography (CT scan) or cardiologic or cerebrovascular tests.

\section{SURVEY OF LITERATURE}

The present work comprises an exhaustive survey of relevant literature of most relevant articles on Soft Computing techniques applied in Neuro Science from reputed journals from IEEE Transactions, Springer and Elsevier publishers and proceedings of international conferences. Some selected articles from this survey are discussed by way of illustration.

Klaus Spitzer, Andreas Thie, Louis R. Caplan and Klaus Kunze [1] (1989) have designed a prototype MICROSTROKE expert system to categorize and diagnose stroke type based on clinical information. The knowledge base of MICROSTROKE includes information from large stroke registries. The system first queries the physician-user for details of the patient's history, information about the onset of stroke, accompanying symptoms and pertinent neurologic findings and then sums the individual data items, factors in the a priori odds, and arrives at the probabilities of different stroke types for a given patient. Stroke type diagnoses by MICROSTROKE were correct in $72.8 \%$ of 250 cases in the Hamburg Stroke Data Bank, stroke types can be of prime importance. Authors presented MICROSTROKE, the prototype of an expert system for computer-supported stroke type diagnosis based only on clinical and historical patient data available at the bedside; MICROSTROKE serves as an aid in the diagnostic work-up of stroke as both a stroke patient data bank and as an educational tool in clinical teaching.

Cruz J, Barahona P, Figueiredo A.P, Veloso M and Carvalho M [2] (1994) have discussed a new knowledge-based system called DARE for the diagnosis of neuromuscular disorders that performs anatomic-physiological reasoning on a deep causal-functional model of the domain knowledge. These characteristics make the system more flexible and general than similar systems in this domain and favour its potential use in different local environments. This paper also discusses the preliminary evaluation of the system performed in the European project ESTEEM, as well as the work still required to make it a real clinical application. The current version of DARE already achieved a quite acceptable diagnostic performance and many improvements can done in the near future such as a need to extend the knowledge model either quantitatively (representing more anatomical structures and functionalities) or qualitatively (including explicitly the etiologic and temporal knowledge) and to support the adopted reasoning methods with more formalized models (allowing the formal definition of reasoning tasks for diagnosis, prognosis and patient treatment).

Christophe S. Herrmann [3] (1995) combined Fuzzy Logic, neural network and an expert system to build a hybrid diagnosis system, a new approach to the acquisition of knowledge bases. This system consists of a fuzzy expert system with a dual source knowledge base. Two sets of rules are acquired, inductively from given examples and deductively formulated by a physician. A fuzzy neural network serves to learn from sample data and allows extracting fuzzy rules for the knowledge base. The diagnosis of electroencephalograms by interpretation of graph elements serves as visualization for author's approach. Preliminary results demonstrate the promising possibilities offered. 
The hybrid system, described in this article, introduces the following new paradigms of modelling the cognitive task of diagnosis, Instead of either acquiring the whole knowledge base automatically from examples, being an inductive learning method.

Leda Cosmides and John Tooby [4] (1997) have authored an article The Cognitive Neuroscience of Social Reasoning. Cognitive scientists needed theoretical guidance that is grounded in something beyond intuition. Authors need evolutionary biology's "adaptationist program", a research strategy in which theories of adaptive function are key inferential tools, used to identify and investigate the design of evolved systems. Using research on how human's reason about social exchange. The authors illustrated how theories of adaptive function can generate detailed and highly testable hypotheses about the design of computational machines in the human mind and reviewed research that tests for the presence of these machines. This research suggests that the human computational architecture contains an expert system designed for reasoning about cooperation for mutual benefit, with a subroutine specialized for cheaper detection. By using a computational theory specifying the adaptive problems entailed by social exchange authors team were able to predict, in advance, that certain very subtle changes in the content and context would produce dramatic differences in how people reason. Authors concluded that the adaptationist program is cognitive neuroscience's best hope for achieving this goal.

Jean-Marc Fellous, Jorge L. Armony and Joseph E. LeDoux [5] (2000) have published the article Emotional Circuits and Computational Neuroscience. Emotion is clearly an important aspect of the mind, yet it has been largely ignored by the "brain and mind (cognitive) sciences" in modern times. However, there are signs that this is beginning to change. Authors discussed some issues about the nature of emotion, describe what is known about the neural basis of emotion and consider some efforts that have been made to develop computer-based models of different aspects of emotion. It is important to distinguish between emotional experiences and the underlying processes that lead to emotional experiences. One of the stumbling blocks to an adequate scientific approach to emotion has been the focus of the field on constructing theories of the subjective aspects of emotion. Studies of the neural basis of emotion and emotional learning have instead focused on how the brain detects and evaluates emotional stimuli and how, on the basis of such evaluations, emotional responses are produced. Computational approaches to emotional processing are both possible and practical. Although relatively few models currently exist, this situation is likely to change as researchers begin to realize the opportunities that are present in this too-long neglected area.

Emmanuil Marakakis, Kostas Vassilakis, Emmanuil Kalivianakis, Sifis Micheloyiannis [6] (2005) have designed Expert System for Epilepsy with Uncertainty, that derives differential diagnosis about epilepsy cases in childhood, according to international classifications. This expert system is intended to be used as a consultation system by neurologists. The physician specialist can update the knowledge base of the system through a user friendly interface. Uncertainty is handled by using weights and certainty factors. Meta-rules drive the reasoning process of the system. The initial evaluation results of the system are very promising, i.e. $83.3 \%$ successful diagnosis. HIPPOCRAT-EES preliminary results are satisfactory compared with the results of the systems. The results from the evaluation of the decision support system in are as follows. The system has derived correct diagnosis in $85.2 \%$ of patient cases, partial successful diagnosis in $8.2 \%$ of patient cases and absolute incorrect diagnosis in $6.6 \%$ of patient cases. The diagnosis of the DSS is more accurate than the one of HIPPOCRAT-EES. On the other hand the DSS does not derive alternative diagnoses as HIPPOCRAT-EES does. In addition, the KB of the DSS cannot be updated by physicians like HIPPOCRAT-EES. The diagnosis of the system has been compared with the diagnosis of three experts. The evaluation results are $72 \%$ correct diagnoses, $8 \%$ partially correct diagnoses and $20 \%$ incorrect diagnoses. 
Blacksmiths, Neurons Mauro Adenzato and Francesca Garbarini [7] (2006) authored the paper Cognitive Science, Neuroscience and Anthropology - A Journey among Robots. In recent years, neurophysiological and psychological research has highlighted a pragmatic version of the theory of knowledge, a version in which the concept of simulation has been found to play a crucial role.

In fact, research on canonical and mirror neurons has shown that as if simulative schema is required to perceive, categorize and understand the meaning of the external world. The present study compares the cognitive paradigm of embodied cognition with Pierre Bourdieu's practice theory. Specifically, cognitive processes and cultural mechanisms are described as phenomena that emerge from the dynamic interaction that exists between people's practical abilities and the structure of the local environment in which they act and live. A pragmatic conception of knowledge has also emerged in the field of ethnological investigation. Indeed, the concepts of resonance and empathy have proven to be essential instruments for ethnological knowledge. With this new view of the relationship between mind and body and between culture and nature, there are now greater opportunities for conducting interdisciplinary research in the natural sciences and social sciences, research aimed at reconstituting the fracture that has existed for too long now between humans as biological and cultural beings. As Jacob and Jeannerod (2005) observed, the mirror neuron system is well designed for representing an agent's motor intention, but not the agent's prior intention to execute an action. A discussion on the theoretical distinction between motor intention and prior intention goes beyond the aims of the present work. Authors referred to Becchio, Adenzato and Bara (2006) for a more exhaustive analysis of the issue.

Badri Adhikari, Md. Hasan Ansari, Priti Shrestha and Susma Pant [8] (2008) have developed a Neurology Diagnosis System, which is concerned about the construction of a web-based expert system. The objective of the system is to help the diagnosis process of neurology doctors. Neurology is a medical specialty that deals with disorders of the nervous system. Doctors will use the website as a helpful tool to diagnose their patients. The web application will collect rules of the neurology domain and cases of the patients. Integrating the techniques of rule-based reasoning and case-based reasoning a hybrid system can be constructed. The system will use the rules and cases to achieve the objective of assisting the decision making process of the domain experts. The proposed system will prove effective, efficient and will establish itself as a valuable asset of the department and the hospital. Precise analysis of return on investment (ROI) and breakeven analysis is difficult at this proposing stage of an academic project. It can be assured that the project will prove economically feasible.

Murad Alaqtash, Huiying Yu, Richard Brower, Amr Abdelgawad, Eric Spier, and Thompson Sarkodie-Gyan [9] (2010) have authored Application of Wearable Miniature Non-invasive Sensory System in Human Locomotion using Soft Computing Algorithm. The authors have designed and tested a wearable miniature non-invasive sensory system for the acquisition of gait features. The sensors are placed on anatomical segments of the lower limb, and motion data was then acquired in conjunction with electromyography (EMG) for muscle activities, and instrumented treadmill for ground reaction forces (GRF). A relational matrix was established between the limb-segment accelerations and the gait phases. This algorithm offers the possibility to perform functional comparisons using different sources of information. It can provide a quantitative assessment of gait function. This algorithm has clearly illustrated the possibility to perform functional comparisons by using different sources of information.

The fuzzy similarity methodology depicts distinctions between the reference able-bodied and the randomized test subjects within with a membership grade of belonging. This novel algorithm may offer very reliable and efficient tools for the evaluation and assessment of gait function in several ways: By building a rule-based system depicts the strength of relation between muscle activities, 
limb-segment accelerations, and gait phases. By comparing the reference muscle activities within gait phases with a randomized input-matrix through a fuzzy similarity algorithm.

Youssouf EL ALLIOUI and Omar EL BEQQALI [10] (2010) have authored the article O'Neurolog - Building, an Ontology for Neurology in Mobile Environment, The (context aware services). A crucial requirement for the context-aware service provisioning is the dynamic retrieval and interaction with local resources, i.e., resource discovery. The high degree of dynamicity and heterogeneity of mobile environments requires rethinking and/or extending traditional discovery solutions to support more intelligent service search and retrieval, personalized to user context conditions. Authors research work aims at providing suitable answering mechanisms of mobile requests by taking into account user contexts (preferences, profiles, physical location, temporal information...). This paper proposes ontology, called O'Neurolog, to capture semantic knowledge a valuable in Neurology domain in order to assist users (doctor, patient, administration ...) when querying Neurology knowledge bases in mobile environment. First, authors designed a domain specific ontology, called O'Neurolog that incorporates concepts drawn from raw data and expert knowledge. In fact, data and knowledge discovery is a crucial activity in pervasive environments where mobile users need to be provided with personalized results due to limited physical characteristics of portable devices. Another interesting future issue authors envision dealing which is the resolution of conflicts that may arise between value or priority preferences. Authors believe that a possible approach may be the definition of meta-preferences, as authors have began the formalization in section.

Faran Baig, M. Saleem Khan, Yasir Noor and M. Imran [11] (2011) have designed model of Fuzzy Logic Medical Diagnosis Control System, This research work addresses the medical diagnosis regarding the normality of a human function in human brain and the diagnosis of haemorrhage and brain tumour. It enhances the control strategies in the medical field to diagnose a disease. The simulation results are found in agreement with the design based calculated results. This research work proposes to develop a control system to enhance the efficiency to diagnose a disease related to human brain. Both the design model and simulation result are same. The designed system can be extended for any number of inputs. Normal, haemorrhage and the brain tumour all depend on the inputs protein, red blood cell, lymphocytes, neutrophils and eosinophils. Authors defined this system for any number of inputs. As the inputs are the blood cells and the designed system use five blood cells as inputs, similarly authors defined this system more than five Inputs to get more efficient human diagnose results. The design work was being carried out to design state of the art fuzzy logic medical diagnosis control system in future using FPGAs.

Dragan Simić, Svetlana Simić, and Ilija Tanackov [12] (2011) authored the article An Approach of Soft Computing Applications in Clinical Neurology, this paper briefly introduces various soft computing techniques and presents miscellaneous applications in clinical neurology domain. Authors presented applications of soft computing models of the cutting edge researches in neurology domain, specifically for EMG and EEG signals. This paper only indicates some researches based on hybrid soft computing and expert and decision support systems. Also, researches on implementation of different artificial intelligence techniques - hybrid soft computing methods can be applied to almost all medical domains, neurology included.

Rajdeep Borgohain and Sugata Sanyal [13] (2011) designed Rule Based Expert System for Cerebral Palsy Diagnosis. The use of artificial intelligence is finding prominence not only in core computer areas, but also in cross disciplinary areas including medical diagnosis. The expert system takes user input and depending on the symptoms of the patient, diagnoses and if the patient is suffering from Cerebral Palsy. The expert system also classifies the Cerebral Palsy as mild, moderate or severe based on the presented symptoms. Authors have discussed the design and implementation of a rule based Expert System for Cerebral Palsy Diagnosis. This expert 
system helps to diagnose Cerebral Palsy and classify it as mild, moderate or severe. In the implementation, authors have taken the most classical symptoms of Cerebral Palsy and given a weight age to each of the symptom and according to the feedback given by the user.

The expert system can go a great deal in supporting the decision making process of medical professionals and also help parents having children with Cerebral Palsy to assess their children and to take appropriate measures to manage the disease.

Imianvan Anthony Agboizebeta and Obi Jonathan Chukwuyeni [14] (2012) have authored Cognitive analysis of multiple sclerosis utilizing fuzzy cluster means Multiple sclerosis, often called MS, is a disease that affects the central nervous system (the brain and spinal cord). Myelin provides insulation for nerve cells improves the conduction of impulses along the nerves and is important for maintaining the health of the nerves. In multiple sclerosis, inflammation causes the myelin to disappear. Genetic factors, environmental issues and viral infection may also play a role in developing the disease. MS is characterized by life threatening symptoms such as; loss of balance, hearing problem and depression. This paper presents a diagnostic fuzzy cluster means system to help in diagnosis of Multiple sclerosis using a set of symptoms. This advanced system which uses clustered data set is more precise than the traditional system. The classification, verification and matching of symptoms to the two groups of clusters (Relapsing/remitting multiple sclerosis and Primary Progressive Multiple Sclerosis) was necessary especially in some complex scenarios. This paper demonstrates the practical application of ICT (Information and communication technology) in the domain of diagnostic pattern appraisal in medicine by determining the extent of membership of individual symptoms. The model proposed allows for the classification of matching of cluster groups to multiple sclerosis symptoms. The fuzzy-cluster means model proposed in this paper appears to be a more useful.

Sujit Das, Pijush Kanti Ghosh and Samarjit Kar [15] (2012) have authored Hypertension Diagnosis: A Comparative Study using Fuzzy Expert System and Neuro Fuzzy System. Hypertension is called the silent killer because it has no symptoms and can cause serious trouble if left untreated for a long time. It has a major role for stroke, heart attacks, heart failure, aneurysms of the arteries, peripheral arterial diseases, chronic kidney disease etc. Then this paper presents a comparative study between fuzzy expert system (FES) and feed forward back propagation based neuro fuzzy system (NFS) for hypertension diagnosis. This paper also presents a comparison among the learning functions (LM, GD and BR) where Levenberg-Marquardt based learning function shows its efficiency over the others. Comparison between FES and NFS shows the effectiveness of using NFS over FES. Here, the input data set has been collected from 10 patients whose ages are between 20 and 40 years, both for male and female. It has shown that the neuro fuzzy system used in this study has the capacity to produce higher overall prediction accuracy than particular fuzzy expert system architecture. Based on this observation authors concluded that NFS represents a useful method for medical diagnostic task of finding hypertension risk factor. Different ANN training algorithms were shown to lead to different diagnostic results among which Levenberg- Marquardt is proved to be optimal. Development of NFS would be more helpful to medical experts and new coming practitioner for diagnosing the hypertension in proper order. For feed forward back propagation based neural network, authors have taken the membership values of related linguistic variables (low, medium and high) as input of age, BMI, BP and Heart Rate and as output (less, moderate and severe) for Hypertension risk evaluation. In this present study, FES shows hypertension risk is moderate for 5 patients and less for the other five patients. But NFS shows severe risk for 5 patients, less risk for 4 patients and moderate risk for 1 patient which is close to medical observation as released by a team of experts. Future goal of this study is to present the adaptive neuro fuzzy inference system (ANFIS) for diagnosis of Hypertension and to make a comparative study with the existing system. More rules and more symptoms might be added in the future research work for more precise diagnosis. 
Vida Groznika, Matej Guida, Aleksander Sadikova, Martin Mozinaa, Dejan Georgiev, Veronika Kragelj, Samo Ribari, Zvezdan Pirtosek and Ivan Bratkoa [16] (2012) have authored the article Artificial Intelligence in Medicine. This paper describes the use of expert's knowledge in practice and the efficiency of a recently developed technique called argument-based machine learning (ABML) in the knowledge elicitation process. Authors are developing a neurological decision support system to help the neurologists differentiate between three types of tremors: Parkinsonian, essential and mixed tremor (comorbidity). The system is intended to act as a second opinion for the neurologists and most importantly to help them reduce the number of patients in the "gray area" that require a very costly further examination (DaTSCAN). Authors strived to elicit comprehensible and medically meaningful knowledge in such a way that it does not come at the cost of diagnostic accuracy. 122 patients were enrolled into the study. The classification accuracy of the final model was $91 \%$. Equally important, the initial and the final models were also evaluated for their comprehensibility by the neurologists. All 13 rules of the final model were deemed as appropriate to be able to support its decisions with good explanations. This paper demonstrates ABML's advantage in combining machine learning and expert knowledge. The accuracy of the system is very high with respect to the current state-of-the-art in clinical practice and the system's knowledge base is assessed to be very consistent from a medical point of view. Authors have also measured the net time involvement of the expert in building a knowledge base for the system. Authors believe ABML saves a significant amount of expert's time and the experts agreed that the process itself felt very natural and stimulating.

Rajdeep Borgohain and Sugata Sanyal [17] (2012) have discussed the implementation of a rule based expert system for diagnosing neuromuscular diseases. The proposed system is implemented as a rule based expert system in JESS for the diagnosis of Cerebral Palsy, Multiple Sclerosis, Muscular Dystrophy and Parkinson's disease. In this system, the user is presented with a list of questionnaires about the symptoms of the patients based on which the disease of the patient is diagnosed and possible treatment is suggested. This system can aid and support the patients suffering from neuromuscular diseases to get an idea of their disease and possible treatment for the disease. Author's presented an expert system for diagnosis of neuromuscular disorders, which is used to diagnose some of the most common neuromuscular diseases i.e. Cerebral Palsy, Muscular Dystrophy, Parkinson's disease and Multiple Sclerosis. The system is a rule based expert system implemented using the Java Expert System Shell using the backward chaining mechanism. The expert system can go a great deal in supporting the decision making process of medical professionals and also help patients with neuromuscular disorders and give an overview of the disease and treatment options.

A.Sh.AMOOJI [18] (2013) has authored the article the application of expert systems in medical diagnosis, which is very interesting and it creates considerable importance system of diagnosis. The proposed system can help doctors and patients in providing decision support system, interactive training tools and expert skills. The system constitutes part of intelligent system for diagnosis of neurological diseases that used in one of the great hospital in Tehran. All of the neurological diseases diagnoses have been investigated in this project. The system constitutes part of intelligent system of diagnosis of neurological diseases. The present expert system is evolving and increasing efficiency for all neurological diseases. Therefore the work was aimed to design a system for the diagnosis of neurological diseases using FC (Fuzzy Cognitive) which is a successful application of Lotfizadeh's fuzzy set theory. It is a reasonable tool for dealing with uncertainty and imprecision and the knowledge of a physician can be modelled using an FC. Usefulness and power of a FC depends on its knowledge base which consists of a data base and a rule base. It is observed that the performance of a FC mainly depends on its rule base, and optimizing the membership function distributions stored in the data base is a fine tuning process. 
The proposed work can be further improved and lengthened for the automation of disease (cancer, heart disease, arthritis) prediction with the help of genetic algorithm and microarray gene expression. Real data from health care organizations and agencies needs to be collected and all the available techniques will be compared for the optimum accuracy.

Vahid Eslami, Sadreddin Rouhani-Esfahani, Nima Hafezi-Nejad, Farshid Refaeian, Siamak Abdi, and Mansoureh Togha[19] (2013). A computerized expert system for diagnosing primary headache based on International Classification of Headache Disorder. The authors developed a computerized program designed to diagnose primary headache based on international classification of headache disorders, criteria for use by physicians. An appropriate questionnaire was designed according to the ICHD-II criteria for all types of primary headaches and the computerized system provided diagnosis based on the criteria. The software was tested by analyzing 80 patients, recruited from an outpatient headache clinic, affected by primary headache. Each patient with a unique card number was interviewed up to 15 minutes. At the end of each day, software and neurologist diagnoses were evaluated for each patient. Of 80 patients, the software was able to come up with correct results in 78 cases. The age of the patients ranged from 30 to 80 years old. Migraine headache accounted for 71 cases, five patients had tension type headache, and 2 had cluster headaches; all were correctly diagnosed by software. Two cases were not concordant with the neurologist's diagnosis. The neurologist diagnosed these two cases as "Medical overuse syndrome headache" and "cluster headache", which authors software was not able to diagnosis them. This software permitted the diagnosis of more than $97 \%$ of the patients similar to the physician's. Authors hope this questionnaire and applying the software to diagnose headache based on ICHD could be of help to better the diagnosis of headaches.

Atul Krishan Sharma and Stuti Gupta [20] (2014) have developed Neurological Disorder Diagnosis System. This paper presents an account of Rule-Based Expert System (RBES) for Neurological Disorders, i.e., Alzheimer, Parkinson, Tetanus disease, Cerebral Palsy, Meningitis, Epilepsy, Multiple Sclerosis, Stroke, Cluster headache, Migraine, Meningitis. Neurological disorders are mainly concerned with the malfunctioning of nervous system. Detection and monitoring of neurological disorders at early stage is essential for quality life and facilitate necessary diagnosis and treatment of the diagnosed disease. The focus of this paper is the development of Neurological Disorder Diagnosis System (NDDS), which can act as home agent to detect the disorder with accuracy to that of an expert. The system consists of a knowledge base with some facts. On the basis of these facts the medical practitioner will fed symptoms as input. The system by applying inference procedures will return the output as results. More than 10 types of neurological diseases can be diagnosed and treated by the system. In this paper, Neurological Disorder Diagnosis System (NDDS) a rule based expert system is developed which helps in diagnosing a nervous system disorder by analyzing the observed symptoms. This expert system is developed to be used as a consultation system for neurologists and researchers in order to reach a decision. The system developed is different from previously developed systems is in terms of accuracy. The system is developed to be near possible as accurate as a human expert. This system can be made advanced to deal with uncertainty using Fuzzy Based Reasoning Techniques. Fuzzy logic provides high accuracy for problems based on uncertainty. The system can also be developed as touch screen systems which can act as pocket systems to detect neurological disorders.

Maíra Junkes-Cunha, Glauco Cardozo, Christine F Boos and Fernando de Azevedo [21] (2014) have authored Implementation of expert systems to support the functional evaluation of stand-tosit activity background, functional evaluation of sit-to-stand and stand-to-sit activities was often used by physiotherapists in patients with neurological and musculoskeletal disorders. The observation of the way these activities are executed is essential in identifying kinesiological problems. 
There are different methodologies used to describe the stand-to-sit activity and its evaluation is not yet standardized, which makes the practical application of resources on clinical observation difficult. The objective of this study is to automate the decision making process of an evaluation protocol, developed in previous study and facilitate its utilization by professionals in the area.

The developed expert systems can support the physiotherapist in evaluating stand-to-sit activity through a conclusion suggestion about the "level of inadequacy" for the "degree of inadequacy" searched during its execution. Results of experts evaluation analyzed through statistical methods indicate that the automation of protocols contributed to the standardization of the evaluation of stand-to-sit activity and that it has application for teaching purposes.

Komal R. Hole and Prof.Vijay S.Gulhane Rule-Based Expert System for the Diagnosis of Memory Loss Diseases [22] (2014).The paper presents a Rule-based Expert System for Memory Loss Disease with the help of rules and facts. Also the Case-based approach is used for saving the cases and for comparing the new case with previously saved cases. It will initially discuss different approaches in designing of Medical Diagnosis Expert Systems with focus on all the information about the memory loss. The different symptoms and causes of memory loss at different age groups and the precautions for any kind of memory loss are covered. It is an attempt to focus on some of very important diseases related to memory loss like Alzheimer's disease, Parkinson's disease, Huntington's disease, and dementia which are among the most common types of memory loss diseases. The rule-based and case-based reasoning can be used for designing diagnostic system. Case-based reasoning is often used where experts find it hard to articulate their thought processes when solving problems. Expert system is a computer program designed to model the ability of solving a problem by a human. In this dissertation an expert system has been introduced to diagnose and suggest the treatments for the type of memory loss diseases. Hence, first the purpose and goals of an expert system were defined and then the relevant research reviewed. The case-based medical expert system prototype that supports diagnosis of common diseases was developed. Several properties of this model remain to be to be investigated. It should be tested on several more databases. Unfortunately databases are typically proprietary and difficult to obtain. Future prospects for medical databases should be good since some hospitals are now using computerized record system instead of traditional paper based. It should be fairly easy to generate data for machine diagnosis.

\section{Conclusions}

This literature survey reveals that many researchers have applied soft computing techniques to neurology problems. At the outset authors concluded that even after having significant research in this field. The practical use of expert system by a neurologist in Indian hospitals is limited. Authors would like to conduct an field survey of this aspect and would like to explore why the use of research tools developed has not been/could not been applied in practice. This survey would help us to understand the difficulties and limitations of such tools/software in Indian context.

Authors would like develop an innovative solution which will suit our Indian neurologist doctors requirements particularly remote and rural areas.

\section{ACKNOWLEDGEMENTS}

The authors would like to express gratitude to NMAM Institute of Technology, Nitte and Basaveshwar Science College, Bagalkot for their support and funding present research. Authors 
would like to thank the Vision Group of Science and Technology, Government of Karnataka for sponsoring the project under K-Fist Level-1 Project Soft Computing Applications in Business and Industry sectors (GRD - 248, 2013-14)

\section{REFERENCES}

[1] Klaus Spitzer, Andreas Thie, Louis R. Caplan and Klaus Kunze (1989), The MICROSTROKE Expert System for Stroke Type Diagnosis, American Heart Association Print ISSN: 0039-2499. Online ISSN: $\quad 1524-4628 \quad$ doi: 10.1161/01.STR.20.10.1353,Stroke.;20:1353-1356 http://stroke.ahajournals.org/content/20/10/1353

[2]. Cruz J, Barahona P, Figueiredo A.P, Veloso M, Carvalho M. UNINOVA (1994), DARE: a Knowledge-Based System for the Diagnosis of Neuromuscular Disorders, Hospital Egas Moniz, Hospital Santa Maria, pp :1-12

[3]. Christophe S. Herrmann (1995) A Hybrid Fuzzy - Neural Expert System for Diagnosis, Pp 494-500, herrmann@intellektik.informatik.th-darmstadt.de

[4]. Leda Cosmides and John Tooby (1997), The Cognitive Neuroscience of Social Reasoning, The New Cognitive Neurosciences Second Edition, A Bradford Book The MIT Press Cambridge, Massachusetts London, England, Pp : 1259-1270

[5]. Jean-Marc Fellous, Jorge L. Armony and Joseph E. LeDoux (2000), Emotional Circuits and Computational Neuroscience, Road Map: Connectionist Psychology Related Reading: Conditioning; Emotion-Cognition Interactions; Sparse Coding in the Primate Cortex, Pp : 1-6

[6]. Emmanuil Marakakis, Kostas Vassilakis, Emmanuil Kalivianakis, Sifis Micheloyiannis (2005) Expert System for Epilepsy with Uncertainty, AIML 05 Conference, 19-21 December 2005, CICC, Cairo, Egypt http://www.cs.teicrete.gr

[7]. Blacksmiths, Mauro Adenzato and Francesca Garbarini (2006), The As If in Cognitive Science, Neuroscience and Anthropology A Journey among Robots and Neurons, Theory \& Psychology, Sage Publications. Vol. 16(6): 747-759 DOI: 10.1177/0959354306070515 www.sagepublications.com University of Turin

[8]. Badri Adhikari, Md. Hasan Ansari, Priti Shrestha and Susma Pant (September 25, 2008 ) "Neurology Diagnosis System", Module

[9]. Murad Alaqtash, Huiying Yu, Richard Brower, Amr Abdelgawad, Eric Spier and Thompson Sarkodie-Gyan (2010), Application of Wearable Miniature Non-invasive Sensory System in Human Locomotion Using Soft Computing Algorithm

[10]. Youssouf EL ALLIOUI and Omar EL BEQQALI (September 2010), O'Neurolog- Building an Ontology for Neurology in Mobile Environment, IJCSNS International Journal of Computer Science and Network Security, VOL.10, No.9,

[11]. Faran Baig, M. Saleem Khan, Yasir Noor, M. Imran, Faran Baig et al. (5 May 2011), Design Model of Fuzzy Logic Medical Diagnosis Control System, International Journal on Computer Science and Engineering (IJCSE) ISSN : 0975-3397 Vol. 3, Pp:2093-108

[12]. Dragan Simić, Svetlana Simić, and Ilija Tanackov (2011), An Approach of Soft Computing Applications in Clinical Neurology E. Corchado, M. Kurzyński, M. Woźniak (Eds.): HAIS 2011, Part II, LNAI 6679, Pp. 429-436, 2011. (C) Springer-Verlag Berlin Heidelberg

[13]. Rajdeep Borgohain, Sugata Sanyal (2011) Rule Based Expert System for Cerebral Palsy Diagnosis, $\mathrm{Pp}: 1-4$

[14]. Imianvan Anthony Agboizebeta and Obi Jonathan Chukwuyeni (January 2012), Cognitive Analysis of Multiple Sclerosis Utilizing Fuzzy Cluster Means, International Journal of Artificial Intelligence \& Applications (Ijaia), Vol.3, No.1, Tripplejo2k2@Yahoo.Com

[15]. Sujit Das, Pijush Kanti Ghosh and Samarjit Kar (2012), Hypertension Diagnosis: A Comparative Study using Fuzzy Expert System and Neuro Fuzzy System, Pp: 1-7.

[16]. Vida Groznika, Matej Guida, Aleksander Sadikova, Martin Mozinaa, Dejan Georgiev, Veronika Kragelj, Samo Ribari, Zvezdan Pirtosek, Ivan Bratkoa(2012), Artificial Intelligence in Medicine Contents lists available at SciVerse ScienceDirect Artificial Intelligence in Medicine journal, No. of Pages 12

[17]. Rajdeep Borgohain, Sugata Sanyal (2012) Rule Based Expert System for Diagnosis of Neuromuscular Disorders, Pp : $1-5$ 
[18]. A.Sh. Amooji (2013) The Expert System for Diagnosis of Neurological Diseases Using Fuzzy Cognitive, Baki Universitetinin Xəbərləri, №3 Fizika-Riyaziyyat Elmləri Seriyası 2013 İnformatika, Pp:95-102, It_Iranian@Yahoo.Co

[19]. Vahid Eslami, Sadreddin Rouhani-Esfahani, Nima Hafezi-Nejad, Farshid Refaeian, Siamak Abdi, And Mansoureh Togha A Computerized Expert System For Diagnosing Primary Headache Based On International Classification Of Headache Disorder, (ICHD-II) Copyright $\odot$ Eslami Et Al.; Licensee Springer. 2013 This Article Is Published Under License To Biomed Central Ltd. (Http://Creativecommons.Org/Licenses/By/2.0)

[20]. Atul Krishan Sharma, Stuti Gupta Neurological Disorder Diagnosis System www.ijraset.com Vol. 2 Issue VI, June 2014 ISSN: 2321-9653, International Journal for research in applied science and engineering technology (ijraset)

[21]. Maíra Junkes-Cunha, Glauco Cardozo, Christine F Boos and Fernando de Azevedo, (2014) Implementation of expert systems to support the functional evaluation of stand-to-sit activity, Bio Medical Engineering On Line 2014, 13:98 http://www.biomedical-engineeringonline.com/content/13/1/98

[22]. Miss. Komal R. Hole, Prof.Vijay S.Gulhane.Rule-Based Expert System for the Diagnosis of Memory Loss Diseases. IJISET - International Journal of Innovative Science, Engineering \& Technology, Vol. 1 Issue 3, May 2014. www.ijiset.com ISSN 2348 - 7968

\section{AUTHORS}

Prof. Dr. D.K.Sreekantha is serving as Professor in the Dept. of Computer Science \& Engineering, NMAM Institute of Technology, Nitte Karnataka. His areas of interest are Soft Computing, Expert Systems, Knowledge Management, Risk Management, Banking, Finance and Business Applications. He has completed two funded research projects

Mr. T.M.Girish is a faculty in the Dept. of Computer Science in Basaveshwar Science College, Bagalkot. His areas of Interest are Soft Computing and Medical Applications

Prof. Dr. R.V.Kulkarni is the Head, Dept. of Computer Studies, CSIBER institute, Kolhapur, 8 students have been awarded Ph.D under his guidance. He has authored more 60 papers in International journals

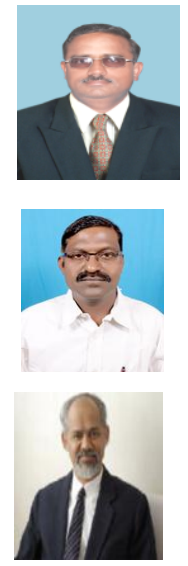

